\title{
EDUCACIÓN TECNOLÓGICA: UNA NUEVA ASIGNATURA EN TODO EL MUNDO
}

\author{
GILBERT, J. K. \\ Departamento de Educación Tecnológica y Científica, Universidad de Reading, Gran Bretaña.
}

\section{SUMMARY}

This paper has three interlocking themes. The first relates to rationales for the inclusion of technology education in the school curriculum worldwide. The second deals with the issues that have to be addressed as that inclusion is either made or extended. The third is the relation between technology and the environment, which has implications for the problems addressed by technology education.

\section{INTRODUCCIÓN}

Durante la última década ha tenido Iugar en muchos países la introducción de la educación tecnológica en los programas generales escolares, ya sea como una asignatura aparte o impartida a través de asignaturas existentes. Esta introducción continúa ampliando su ámbito de provisión. Debe de considerarse que tiene mucho que ofrecer a los estudiantes, ya que en todas las escuelas se disputan fieramente los horarios, las asignaciones de personal y otros recursos. ¿Qué argumentos se esgrimen a su favor? ¿Qué temas estratégicos condicionarán que se acepte mundialmente? ¿Qué temas tácticos controlarán su establecimiento en cualquier sistema de educación nacional?

Es particularmente difícil contestar a estas preguntas porque, si no existe una definición de la tecnología que sea universalmente aceptada, mucho menos habrá una para la educación tecnológica. La tecnología tiene dos amplios significados. Por un lado constituye la suma de conocimientos y capacidades que se utilizan en el proceso de solucionar problemas prácticos que son importantes para la humanidad, por ejemplo, la provisión de una vestimenta adecuada. Por otro lado también representa los objetos o sistemas que son producto de estos esfuerzos, por ejemplo, la ropa producida. La tecnología, como la suma de proceso y producto, es tan antigua como la humanidad. Otras especies muestran comportamientos que se pueden describir como prototecnológicos, como es el uso de piedras para romper cáscaras. Pero en su forma avanzada debe figurar, junto con el desartollo de Ia lengua, como uno de los mayores logros de la huma- nidad La relación entre la tecnología y la educación tecnóógica constituye el núcleo de este estudio.

\section{LOS ARGUMENTOS, ACERCA DE LA EDUCACIÓN TECNOLÓGICA}

Desde el punto de vista práctico, se presentan tres conjuntos de motivos: económicos, sociales y educativos (Medway 1989, Layton 1993).

Los argumentos económicos se centran en la importancia de la tecnología para las actividades creadoras de riqueza de todos los países. Los que están a favor de la educación tecnológica en las escuelas defienden la educación prevocacional: la preparación de los jóvenes para el mundo laboral. Se dice que la inclusión de este elemento no sólo aumentaría las posibilidades de que los jóvenes que acaban la escolaridad obtuviesen trabajo en la industria, sino que además se espera que los predispondría para este trabajo. Los que están en contra señalan la naturaleza transitoria del empleo en una industria clatamente basada en la tecnología y quieren ver el énfasis en capacidades básicas (leer, escribir, aritmética) y en conocimientos valorados socialmente, como la historia nacional, la geografía y la cultura.

Los argumentos sociales adoptan dos formas. Primeramente, se razona que la toma de decisiones personales, econórnicas y sociales, especialmente en sociedades 
democráticas, requiere que personas privadas, industriales y legisladores sean conscientes de sus acciones relacionadas con la tecnología. En segundo lugar, dado que la mitigación de las consecuencias no deseadas de la tecnología del pasado también necesitará, de por sí, el uso de la tecnología en el futuro, se necesita Ja experiencia y las capacidades apropiadas en esta área.

I.os argumentos educativos se desglosan en la siguiente serie de puntos. En primer lugar, si la tecnología es uno de los supremos logros de la humanidad, entonces todos los jóvenes deberían entrar en contacto con ella. En segundo lugar, teniendo en cuenta que los objetos producto de la tecnología se encuentran en todos los hogares y lugares de trabajo, se debería incluir una introducción a aquéllos y su uso en la preparación para la vida adulta (Silverstone 1991). En tercer lugar, la educación tecnológica se considera un vehículo valioso a través del cual se pueden conseguir fines educativos. Este útimo punto, que parece contener muchos de los argumentos anteriores, merece ser ampliado.

Antes de hacerlo, hay que introducir la visión general de la práctica de la tecnología que se debe a Pacey (1983). Él considera que la tecnología tiene tres aspectos: el aspecto técnico, que se ocupa de los conocimientos y capacidades que se utilizan, junto con materiales tales como metales o enzimas para diseñar y hacer nuevos productos; el aspecto cultural, que se ocupa de los valores subyacentes de la elección de problemas y necesidades que se pretenden solucionar por medio de la tecnología y de los criterios utilizados para valorar los resultados de la opción elegida; el aspecto organizativo, que se ocupa de la economía y la sociología del comportamicnto de la tecnología y de la utilización de sus resultados.

El objetivo más generalizado de todos los sistemas educativos es el desarrollo de todas las capacidades de una persona. ¿Cómo hay que enfocar dichas capacidades? Gardner (1985) ha propuesto que todos los humanos poseen seis inteligencias bastante diferentes. La inteligencia lingüística se ocupa de la fonología, la sintaxis, la semántica, y la práctica de uso cotidiano, de tas lenguas que usa un individuo. La inteligencia musical se ocupa de la comprensión del tono, del ritmo y del timbre $\mathrm{cn}$ la música. La inteligencia lógico-matemätica se utiliza en actividades matemáticas o científicas. La inteligencia espacial se ocupa del reconocimiento de objetos y de la habilidad de manipularlos y transformarlos mentalmentc. La inteligencia corporal-cinestésica se utiliza para el delicado control del movimiento. Y por ser la última pero no la menos importante, la inteligencia personal, que se usa en el desarrollo de una noción de la propia persona construida con el uso de ta inteligencia interpersonal e intrapersonal. Cada una de las inteligencias está sujeta a una corriente de desarrollo durante la primera etapa de la vida, al mismo tiempo que, de vez en cuando, tiene lugar una racha de desarrollo integrado por muchas, si no todas -las siete-.

La posible contribución de la educación tecnológica a la educación general se puede concebir partiendo de la estructura de Gardner ( 1985 ) bajo los aspectos del enfoque de Pacey (1983). «La educación en la tecnología» (Gilbert 1992), la concepción más amplia de la eđucación tecnológica y una que implicaría tratar los tres aspectos de Pacey (1983) sobre la tecnología, facilita el desarrollo de todas las inteligencias de Gardner (1985), excepto la musical a menos que se tomen medidas para incluirla. "La educación sobre la tecnología», que se centraría en los aspectos culturales y de organización de la tecnología, sólo facilitaría el desarrollo de las inteligencias personales y linguísticas. «La educación para la tecnologias, que se centraría solamente en el aspecto técnico de la tecnologia y que -como veremos más adelante-es la forma que más se practica en las escuelas, facilitaría principalmente el desarrollo de Ias inteligencias linguísticas, lógico-matemáticas, espaciales y corporal-cinestésicas. El alcance de la educación tecnológica proporcionada, en términos de Pacey (1983), regirá de esta forma la aportación hecha a la educación general en términos de Gardner (1985).

\section{EL ENFOQUE TECNQLÓGICO DE LA EDUCACIÓN TECNOLÓGICA}

Sea cual fuere el alcance de la educación tecnológica proporcionada en un país concreto, deberá tratar un conjunto de problemas que son de importancia para la humanidad. ¿Cómo se puede llegar a dicho conjunto? Una aproximación histónica seleccionaría un conjunto que hubiese sido de gran importancia en el pasado y para el cual se hubiese ideado y puesto en práctica una solución satisfactoria. Tanto para profesores como estudiantes, no se trataría de problemas reales; con toda probabilidad, se adoptaría un modelo de transmisión de enseñanza, como en casi toda educación formal. Una aproximación contemporánea selecciotraría un conjunto de problcmas que se habrían reconocido como importantes unos años atrás, de forma que las soluciones habrían sido ideadas por la industria y la sociedad y estarían en práctica en la actualidad. Esta aproximación permitiría cierto margen para solucionar problemas reales en las escuelas y podría atraer la participación activa de los estudiantes. Una aproximación orientada hacia el futuro seleccionaría un conjunto de problemas que aún se están formulando: todavía no se han producido soluciones y mucho menos puesto en práctica. El adoptar esta aproximación situaría a las escuelas, al menos en principio, cerca de la primera línea deI avance tecnológico. En la práctica, los sistemas educativos probablemente adoptarían una mezcla de las tres opciones. Pero, dado que es el más estimulante para la planificación educativa, me centraré en la opción de orientación futura.

Las discusiones con los jóvenes muestran que un conjunto de temas, que para ellos son de gran importancia, se centran en el medio ambiente, en formas de reconciliar la conservación con el mantenimiento (jo el avance!) de un nivel de vida adecuadamente alto. Tolba (1992) ha identificado una serie de temas medioambientales, un enfoque que implicaría la tecnología, como proceso y como producto. Éstos son la necesidad de: 
* reducir la contaminación del aire urbano;

* frenar la disminución del ozono atmosférico;

* reaccionar adecuadamente ante los cambios climáti$\cos$;

* aumentar la disponibilidad de agua dulce;

* eliminar las fuentes de polución marina basadas en la tierra;

* invertir la desertización de tierras áridas;

* detener la despoblación forestal;

* frenar la destrucción del hábitat de las marismas;

* detener la pérdida de la diversidad biológica;

* prevenir los desastres naturales causados por el hombre;

* controlar el vertido seguro de residuos peligrosos;

* y, el más importante de todos, detener e invertir el crecimiento de la población humana.

El propósito subyacente en el tratamiento de estos temas debe ser el conseguir una «equidad intergeneracional»: la noción de que una generación humana tiene el uso de la Tierra durante su vida y no debería comportarse de forma que se perjudique el entorno para las generaciones venideras. Esto se tiene que reconciliar con la demanda de niveles de vida más altos, que lleva implícito el trabajo, el principal origen de disciplina social en la mayoría de países. Esta reconciliación es un problema importante, sino el mayor, que sólo actualmente se empieza a tratar seriamente en el ámbito político. La adopción del «desarrollo sostenido" podría ser una noción clave que se ve como:

«...no un cstado fijo de armonía, sino más bien como un proceso de cambio en el que la explotación de los recursos, la dirección de las inversiones, la dirección del desarrollo tecrológico y los cambios institucionales se hacen consistentes con el futuro y también con el presente...»

$$
\text { (W.C.E.P. } 1987, \text { p. 8) }
$$

Obsérvese la importanciá de la tecnología, y en consecuencia la de la educación tecnológica, para conseguir un desarrollo sostenido y para tratar los temas identificados por Tolba (1992). De todas maneras, está cada día más claro que el actual concepto que se tiene de la tecnología como proceso necesitará cambios. Según Juma y Sagoff (1992), la tecnología que mantiene el insostenible desarrollo actual:

* se apoya en una visión de la naturaleza que la considera reducible a entidades sostenidas por conexiones causales determinísticas en un equilibrio que es esencialmente reversible, en el sentido de que se puede normalizar después de grandes distorsiones;
* se basa en una metáfora de la evolución que considera a cada individuo enzarzado en una competición con todos los demás individuos para alcanzar una destacada ventaja cconómica global;

* Ve la tecnología como una propiedad privada preocupada por conseguir el máximo de eficacia, normalmente recmplazando las tecnologias existentes;

* considera que la nueva tecnología se introduce en su forma completa, inmediatamente y sin la debida adaptación ni negociación con los intereses existentes;

* no controla ni considera las consecuencias medio ambientales y sociales de la introducción de una tecnología;

* $\quad c s$ insensible a diferencias de sexo en el diseño y uso de una tecnología.

Por otro lado, una tecnología que da apoyo al desarrollo sostenido:

* se basa en una visión de la naturaleza que se percibe compuesta de entidades que son incapaces de estar completamente separadas unas de otras; éstas se mantienen en un equilibrio irreversible porque la naturaleza no volverá fácilmente a la normalidad después de una gran distorsión; las conexiones entre las entidades son probabilísticas;

* se apoya en una metáfora de la evolución que considera a los individuos comprometidos en una colaboración mutua para conseguir una ventaja económica óptima y locat;

* ve la tecnología como una propiedad colectiva, que persigue una eficacia compatible con el mantenimiento de la sociedad existente y que se integra a las tecnologías existentes.

* considera la tecnología como parcial y progresivamente introducida a través de negociaciones y la adaptación a los intereses exiștentes;

* cree que el control de la tecnología debería ser una requisito legal, por ejemplo, prohibir la producción de subproductos no utilizados.

* es sensibic a las diferencias de género $\mathrm{cn}$ el diseño y uso de tecnologías.

\section{TEMASESTRATÉGICOSEN LA EDUCACIÓN TEC NOLÓGICA MUNDIAL}

Existe una cierta cantidad de temas que se han de tratar cuando la educación tecnológica se introduce en el sistema educativo o se amplía dentro de él. Estos temas son los que se citan a continuación. 


\section{La noción de la tecnología adoptada}

La educación tecnológica aparentemente es una asignatura nueva en el programa escolar, no tiene un claro fundamente teórico derivado de la misma tecnología. Aún no existe una fílosofía coherente de la tecnología en la que basarse.

Se pueden adelantar seis motivos de esa curiosa anomalía que se manifiesta en la falta de departamentos de tecnología, como tales, en las universidades (Margolis 1984, Mackay 1991, Rapp 1989):

* La historia occidental de las ideas ha estado dominada por la teoría, de forma que las actividades prácticas -así es como se ha visto la tecnología- han sido infravaloradas socialmente. La reflexión sobre la tecnología no ha tenido lugar con suficiente frecuencia.

* Existe una ambivalencia social acerca de la tecnología. Hay una visión «humana» que la ve como una respuesta evolutiva a las necesidades humanas, una visión "titánica» que la considera como un intento de subyugar la naturaleza; y una visión «satánica» que la considera social y ambientalmente destructiva. Esta ambivalencia ha paralizado, más que ha estimulado, la discusión y la toma de decisiones.

* Los cambios de la tecnología parece que tienen lugar como respuesta a fuerzas económicas de mercado o bien bajo un estricto control social. La coincidencia de esta perspectiva con puntos de vista políticos ha polarizado la discusión.

* Hasta la Revolución Industrial de los siglos xviil y xix, la tecnología no se veía como una actividad diferenciada. No ha transcurrido el ticmpo suficiente para que se desarrollara una tradición histórica en el mundo académico.

* La tecnología se considera una ciencia aplicada y no una actividad que merece ser estudiada aparte. Han predominado las prioridades científicas establecidas.

* Las actividades de la tecnología no se simplifican con facilidad ni se expresan simbólicamente, to cual es un requisito previo para los estudios avanzados en las universidades tal como se entienden actualmente.

En todas las sociedades que consideren la introducción de la educación tecnológica, tendrán que adoptar un solo punto de vista sobre la tecnología, de las que se han mencionado anteriormente y esto supondrá tres posibilidades. Primero, puede haber un intento de rechazar socialmente la tecnología en sí porque se la considera, al fin y al cabo, intrínsecamente destructiva de una valiosa norma social, que podría ser, por ejemplo, de naturaleza religiosa. En segundo lugar, puede predominar la tecnología para un desarrollo no sostenible. Éste es el caso de muchos países considerados como desarrollados, lo cual lievaría a un limitado punto de vista de la «educación para la tecnología». En tercer lugar, se puede adoptar el punto de vista de la tecnología para un desarrollo sostenible: hasta el momento hay pocos ejemplos de esta última a gran escala. Esto probablemente Ilevará a la adopción de un enfoque global para la «educación en tecnología».

Esta resolución influirá mucho sobre el alcance de la educación tecnológica que se introduzca. También influirá la validez de la educación tecnológica ofrecida. Es esencial que, donde predomine Ja tecnología del punto de vista de desarrollo no sostenible, la educación tecnológica ofrecida se base en un estudio de la verdadera práctica de la tecnológica en la industria. A menos que sea así, existe un grave riesgo de que se proporcione alguna parodia de conveniencia, como es evidentemente el caso en relación con la metodología científica tal como se representa en la educación científica (Millar y Driver 1987). La relativa novedad del punto de vista de «una tecnología para el desarrollo sostenible» sugiere que su verdadera naturaleza necesita una considerable cantidad de razonamiento en su puesta en práctica.

Dentro de cualquiera de los puntos de vista de la tecnología que se adopten habrá otra dimensión: el nivei de tecnología adoptado por la sociedad. En muchos países no todo el mundo puede conseguir los requisitos básicos de la vida. Laa adquisición de comida adecuada, de una vivienda y ropa durables, de unos cuidados sanitarios básicos, de comunicaciones fiables serían el centro de las actividades tecnológicas en esta situación. Donde las economías están algo más avanzadas, las ctecnologías adecuadas» (Budgett-Meakin 1992) podrían convertirse en el centro de atención. En países desarrollados, las «altas» tecnologías de la producción en masa convencional constituyen el fondo mientras que las tecnologías "clave» (Meake 1989) proporcionarían una oricntación futura.

La relación de la educación científica con la educación tecrológica

La relación entre la educación cientifica y la educación tecnológica en cualquier sociedad se ve controlada por la percepción que se tiene de la relación entre las actividades originarias de la ciencia y la tecnología. Un punto de vista es que el comportamiento de la ciencia y de la tecnología son simplemente ejemplos de la aplicación de un modelo general para solucionar problemas (Schilling 1988, Fig. 1). De todas maneras, parece demasiado simple (Gardner et al. 1990, Gilbert 1992). La figura 2 establece otra forma de enfocar las diferencias entre ciencia y tecnología.

No cabe duda de que la relación es estrecha. La ciencia a menudo se divide en ciencia pura, ciencia estratégica y ciencia aplicada. La última se halla más cerca que la primera de muchas formulaciones tecnológicas. El verdadero punto de vista adoptado en un país cualquiera dependerá de la historia institucional de sus principales entidades profesionales gubernativas para la ciencia y la tecnología. 
Figura I

Ciencia y tecnología como ejemplos de solución de problemas.

Modelo general de solución de problemas

Fintender el problema

Describir el problcma

Considerar soluciones alternativas

Elegir la solución

Actuar

Evaluar el producto

\section{Proceso científico}

Fenómeno natural

Describir el problema

Sugerir hipótesis

Seleccionar hipótesis

Experimentar

Encajar hipótesis/datos

\section{Proceso tecnológico}

Determinar la necesidad

Describir la necesidad

Formular idcas

Seleccionar ideas

Hacer el producto

Probar el producto

Figura 2

Diferencias entre la ciencia y la tecnología.

\begin{abstract}
Ciencia
Proposito: Explicación

Interés: Lo natural

proceso: Analítico

Procedimiento: Simplificación del fenómeno

Resultado: Conocimiento generalizable
\end{abstract}

\section{Tecnología}

Propósito: Fabricación

Interés: I o artificia!

Proceso: Sirtético

Procedimierto: Aceptar la complejidad de la necesidad

Resultado: Objeto particular
Sea cual fuese la distribución adoptada en un contexto dado, la ciencia y la tecnología están estrechamente relacionadas. Por lo tanto:

$\because \quad$ Los conceptos de la ciencia se utilizan en la tecnología, aunque sea después de que se haya reducido el nivel de abstracción de conceptos individuales. Los conceptos de las distintas áreas de la ciencia se combinan entre sí para cubrir las necesidades de los problemas que soluciona la tecnología, y se redefinen los conceptos individuales para tratar los contex tos de la tecnología. (Layton $1988,1990 a, b)$.

* La metodología científica se usa para hacer averiguaciones concretas, los resultados de las cuales se necesitan para llevar a cabo la tecnología con éxito.

* Los instrumentos producidos por la tecnologia, tales como los espectrómetros, se usan en la ciencia.

La complejidad de esta relación entre la ciencia y la tecnología se ve reflejada en la gama actual de interacciones entre la educación científica y la educación tecnológica en las aulas; por ejemplo:

: Se hace una referencia mínima a los productos tecnológicos durante las clases de ciencias.
* La enseñanza de conceptos científicos se sigue con una ilıstración de cómo se pueden utilizar en la tecnología.

* La enseñanza de la ciencia se empieza con el análisis de un producto tecnológico concreto.

: Los procesos y productos de la tecnología y de la ciencia sc mezclan durante la enseñanza.

* La relación entre ciencia, tecnología y sociedad se enseña explícitamente, con el énfasis sobre diferentes aspectos de las tres (Gilbert 1992).

La formulación exacta a la que se llegue en cualquier país dependerá de las relaciones entre la comunidad de la enseñanza científica y la de la enseñanza tecnológica: cuanto más cerca estén, más estrechamente relacionada estará la enseñanza.

La historia del plan de estudios de la educación tecnológica

No cabe duda de que la educación tecnológica se está introduciendo en los sistemas educativos de muchos 
países o es reformulada dentro de ellos (Blandow y Dyrenfurth 1992). El análisis basado en la noción de las inteligencias de Gardner ( 1985 ), expuesto anteriormente, sugiere que la mayor contribución de la educación tecnológica a la educación general se haría con la adopción del enfoque de «educación en tecnología». Pero el inherente conservadurismo de los sistemas educativos en la mayoría de países hace que esto sea una aspiración muy alta: la naturaleza de los currículos previos siempre tendrá un impacto sobre la naturaleza de los futuros.

Tal como han señalado tanto Eggleston (1992) como Layton (1993), la historia de la educación tecnológica ticne tres raices, que son:

* una capacidad desarrollada sólo en la educación artesanal, es decir, de forma elemental según el aspecto técnico de Pacey (1983);

* una provisión que cra segregada rígidamente en líneas de género; los varones hacían metalisteria y carpintería y las hembras aprendían cocina (ciencias domésticas) y otras labores del hogar;

* una provisión ideada para los estudiantes menos capacitados.

La forma en que se consolida esta historia de los planes de estudios (o la forma de alejarse de ellos, según el punto de vista) variará de país a país. Además de las variaciones en lo que llamarć "visión curricular», el pragmatismo de las capacidades de los profesores y los recursos físicos y de abastecimiento cobrarán una gran importancia en la toma de decisiones. Muchos países parecen estar muy seguros de la provisión de una «educación para la tecnología» de base amplia. Como mínimo, cubre las demandas de los industriales de una provisión prevocacional en las escuelas. La provisión actual en Gran Bretaña es de esta clase (Eggleson 1992, Gilbert 1992, Layton 1993). La provisión actual en EEUU es también de este tipo (Layton 1993), aunque las aspiraciones hacia ura «cducación en tecnología» son considerables (AAAS 1989). Unas primeras versiones de prescripción en Nucva Zelanda (Jones y Carr 1993) y en Australia (AGPS 1992) sugieren que hay una considerablc ampliación de las perspectivas, aunque eI aspecto organizativo de Pacey (1983) parece cstar más bien poco representado.

\section{Los problemas del género}

La tradicional división de la educación artesanal según el género no fue casual. Se basaba en un modelo dc aprendizaje que pretendía preparar a los jóvenes para papeles sociales que estaban claramente diferenciados por el género. Esta diferenciación de papeles se ha visto considerablemente reducida en la mayoría de países desarrollados durante los últimos cincuenta años aproximadamente, aunque el cambio no está uniformemente distribuido por toda la población. Cualquier país que quiera enmendar la naturaleza de la educación tecnoló- gica que se proporciona en las escuelas lo hará contra una visión general de la sociedad sobre la conveniencia de tales cambios.

Durante los últimos años se ha escrito mucho sobre las cuestiones de género en asuntos de tecnología, afirmándose que:

* los hombres ocupan la mayor parte de posiciones de poder en las industrias productoras de tecnología y, por lo tanto, deciden qué problemas hay que solucionar, cómo se solucionan y qué constituye un producto aceptable;

* las mujeres son los principales usuarios de productos tecnológicos tanto en paises desarrollados como en países en desarrollo, pero tienen un papel muy reducido en la creación de dichas tecnologías;

* los hombres se han asociado al uso de tecnologías relacionadas con el control, la producción, la exploración y el armamento, mientras que las mujeres se han asociado al uso de tecnologías sostenedoras de la vida o de mejora de la vida en las áreas de la sanidad, la alimentación, los textiles y el hogar;

* cl lenguaje utilizado para hablar de la tecnología, las instituciones en las que se habla de ella, y que constituye un argumento adccuado están controlados por la perspectiva masculina.

La división de la tecnología según el género ha tenido un importante impacto negativo sobre la percepción femenina de la educación tecnológica y la voluntad de entrar en ella. Los países que deseen involucrar a las mujeres en la educación tecnológica de forma más general tendrán que considerar las implicaciones de estos puntos en la forma en que se dirige, se ve y se trata la tecnología.

\section{El enfoque para el desarrollo del curriculo}

Hay una historia general sobre la educación tecnológica de alcance limitado. En las útimas décadas ha habido un considerable crecimiento del interés político por este tema. Estos dos factores, considerados conjuntamente, han llevado recientemente a poner el Énfasis sobre la producción de prescripciones nacionales para la «nueva» asignatura, es decir para su iniciación. Éstas $\mathrm{sc}$ distribuyen en las escuelas con la esperanza de que su puesta en práctica tendrá lugar de forma similar. Es posible, utilizando las ideas de Fullan (1991), identificar los elementos de la buena práctica que funcionarían en estas dos fases.

Para la fase de iniciación, que tendria lugar de forma centralizada, como puede ser en una entidad gubernamental responsable de la cducación, es importante que:

* se hayan llevado a cabo los pertinentes proyectos pilotos de alta calidad y que el grupo de planificación conozca los resultados; 
el grupo de planificación csté compuesto por aquéllos que tienen un interés profesional legítimo para estar en él; ciertamente debería tener profesores de educación tecnológica, industriales que practican la tecnología y que están preocupados por la formación del personal industrial, académicos que han estudiado la educación tecnológica así como la tecnología, y administradores partidarios de la innovación. Podrían incluirse otros grupos, como pueden ser las asociaciones profesionales de científicos;

* se llegue a una clara base filosófica que sostenga el currículo, aunque scrá difícil porque las divergentes perspectivas de los intereses representados en el grupo de planificación tendrán que coincidir si se quiere producir una prescripción coherente;

: las discusiones del grupo de planificación incluyan discusiones sobre las implicaciones financieras que ello comporta.

Los burócratas son propensos a pensar que, una vez lanzada, Ia prescripción scrá rápida e jdénticamente puesta en práctica en todas las escuclas. Nada más lejos de la realidad. Las escuelas diseñarán sus provisiones de planificación escolar y los profesores las llevarán a cabo según las experiencias previas en su ámbito: según lo que consideren posible conseguir de forma reatista.

De todas maneras, la prescripción se puede enmarcar de forma que se consiga un alio grado de homogeneidad en la puesta en práctica. Esto ocurrirá si:

* representa una necesidad real por parte de los profesores, padres y, si me atrevo a ser tan radical como para sugcrirlo, los estudiantes;

* tiene una filosofía clara;

$\therefore$ es simple, se considera de alta calidad y bastante compatible con las prácticas de clase existentes.

Es axiomático que una prescripción radical, una que intente alejarse mucho de la norma de las prácticas existentes (en contraposición a la mejor), no será compatible con la práctica habitual. Por lo tanto, es vital que la fase de puesta en práctica esté precedida de, asociada a, y seguida de un programa de reciclaje de desarrollo del profesorado. Bell y Gitbert (en prensa) han presentado un modelo para el desarrollo del profesorado basado en un programa de mucho éxito en Nueva Zelanda, que tiene tres fases o situaciones claramente discernibles:

Sitzación 1. Confirmación. En la situación de confirmación, los profesores han de establecer o reforzar su propia estima desde el punto de vista personal, establecerse dentro de un grupo, sentirse valorados y accplados, y recibir una evaluación como profesionales competentes y como profesores.

Situación 2. Exploración. Desde el punto de vista personal, en la situación de exploración, examinan los sentimientos asociados a ser profesores y a un cambio. En el aspecto social, comprueban la red de apoyo del grupo para establecer los límites y las clases de confianza en el grupo. En el aspecto profesional, los profesores han de considerar y reflexionar sobre nuevas ideas, experimentar en clase con nuevas actividades de enseñanza y aprendizaje, tener más iniciativa en adaptar los materiales para su propio uso.

Situación 3. Adquisición de poder. Desde una perspectiva personal, cn una situación de adquisición de poder, los profesores sacan fuerzas a través de saber quiénes son como profesores y de la confianza, convicción, propiedad y fe en sus tdeas y creencias. En el aspecto social, establecen nuevas situaciones y buscan nuevas agrupaciones para apoyar y continuar su desarrollo personal. Desde el punto de vista profesional, muestran ser competentes en clase con nucvas estrategias, ser realistas, establecer las limitaciones y las ventajas de las nuevas actividades.

Juntamente con la puesta en práctica de una prescripción valiosa, esta actitud ante el desarrollo del profesor debería conducir a lo que Fullan (1991) llama la toma de iniciativa y adquisición del poder deł profesor; a la construcción de visiones; a la reestructuración de programas; a la planificación evolutiva.

\section{ASUNTOS TÁCTICOS DE LA EDUCACIÓN TECNOLOGICA EN EL ÄMBITO NACIONAL}

Además de estos cinco temas estratégicos, existen unos cuantos más que sería mejor considerarlos en cl ámbito nacional. Son los que se citan a continuación.

\section{Proporcionar y formar profesores}

Dada la falta de un estudio coherente de la tecnología en educación secundaria, actualmente hay en la mayoría de paises una sola fuente de posibles profesores de tecnología. Estos profesores en formación se reclutarán entre personas que tienen calificaciones en una de las asignaturas que a menudo contribuycn al aspecto técrico de la educación tecnológica, tales como la artesanía de materiales (carpinterfa, metalistería), economía doméstica (a veces llamada cocina o ciencia doméstica), economía (una ramificación de estudios empresariales), diseño (un sector de las artes), información tecnológica (a veces llamada estudios informáticos). Otros tendrán calificaciones en una de las múltiples ramas de la ingeniería, como la electricidad y la electrónica, la mecánica, los estudios marítimos, producción o química. Muchas de estas personas, sino todas, habrán tenido alguna experiencia industrial, lo cual scrá una ventaja importante para proporcionar una educación tecnológica válida y realista en las escuelas. Es esencial que en su formación antes del servicio se identifiquen y consoliden aquellos clementos de experiencia que sean importantes para la educación tecnológica. 
La formación permanente se deberá impartir para aquellos profesores que hayan desarrollado una experiencia en una o dos de las asignaturas correspondientes. Mientras que habrá elementos de sus conocimientos y de su experiencia en la clase que considerarán indispensables para la nueva asignatura, y que se deberían reconocer en la nueva prescripción, su principal cometido será adquirir los nuevos conocimientos.

E1 equilibrio entre las distintas procedencias de los profesores dependerá de los contex tos nacionales. Cuando tomen parte de cursos de formación inicial y permanente los profesores deberán:

* familiarizarse con la nueva prescripción del currículo, para que puedan ver qué relación guarda con su experiencia anterior, ya sea industrial o como profesores;

* adquirir una amplia gama de conocimientos de fondo, incluyendo conocimientos de la naturaleza de los materiales (madera, metales, plásticos, papel, yeso), la energía y su uso, la teoría de sistemas;

* adquirir, o desarrollar más a fondo, las complejas capacidades de «diseñar y hacer»;

* desarrollar las habilidades apropiadas para el diseño de programa y capacidad de enseñanza. Éstas se tratarán más adelante.

Teniendo en cuenta el amplio ámbito de experiencias de las personas en cuestión y la falta de tiempo y recursos disponibles para su formación, además del amplio ámbito de la posible educación que necesiten, se pueden usar extensamente las técnicas de aprendizaje flexible (Dillon y Davies 1993).

\section{Recursos físicos}

La mayoría de prescripciones actuales para la educación tecnológica incluye los aspectos técnicos de Pacey (1983). Se necesitarán talleres especiales si se da el caso. La provisión de instalaciones de talleres para los estudiantes no está al alcance de los medios financieros de muchos países. Si ya existen, es probable que se hubiesen desarrollado para un fin específico, como la carpintería o las ciencias domésticas. Si una prescripción nacional adopta un punto de vista amplio del aspecto técnico, será necesario o bien transformar todos los talleres para uso general, lo cual puede ser difícit por los rígidos requisitos de la higiene alimentaria, o bien usar instalaciones especializadas con un sistema rotativo. En cualquier caso, dada la provisión tan poco universal de la tecnología en la mayoría de países, de alguna manera se tendrán que proporcionar más talleres.

EI mundo real del diseño cada vez más utiliza el diseño asistido por ordenador (CAD). Si la educación tecnológica se ha de parecer de alguna forma a las técnicas modernas, se necesitarấn amplias instalaciones informáticas. Se argumentará sin duda que las instalaciones de dibujo técnico son adecuadas desde un enfoque histórico para la educación tecnológica.

\section{Diseño y provisión del curriculo}

La introducción en una escuela de una prescripción nueva, o considerablemente revisada, para la educación tecnológica representa un reto importante para todos sus profesores. El error a veces se comete al presuponer que la única capacidad desarrollada en la tecnología de una escuela está dentro de las asignaturas componentes, como las ciencias domésticas. Esto es raramente cierto porque muchos de los elementos de una prescripción amplia ya se enseñan en otras asignaturas. Por ejemplo, los programas de historia contienen a menudo un tratamiento extensivo de los impactos sociales y económicos de la tecnología que se ha introducido en el pasado. El primer paso hacia un cambio importante del plan de estudios ha de ser el realizar una auditoría de la provisión tecnológica existente en la escuela (Davies, Gilbert y Dillon 1991). Utilizando la observación en la clase, entrevistas y cuestionarios, este proceso ticne la finalidad de identificar aquellos elementos de la nueva prescripción tecnológica que ya se están impartiendo, en qué parte del programa, de qué forma y para qué finalidad aparente.

Los resultados consolidados de dicha auditoría, que se encuentran dentro de la tradición de la investigación acción, serán de una utilidad primordial al decidir la estrategia optima para la introducción de una nueva provisión de plan de estudios. Hay tres enfoques que se pueden adoptar: el enfoque coordinado, en el cual las contribuciones separadas de departamentos temáticos se dirigen según un plan general, aunque la provisión tecnológica no tenga un horario propio; cl enfoque modular, en el cual la asignatura de la tecnología se imparte desde varios recursos de personal pero bajo una asignación de horario determinada; el enfoque integrado, en el cual la tecnologia se establece como un departamento temático aparte dentro de la escuela. Cada uno de estos enfoques tiene sus ventajas y desventajas en términos de la efectividad de la provisión y el uso de personal y otros recursos. En cada caso, la solución que será más eficaz a la larga será aquélla en que la provisión de la tecnología más se parezca a la de cualquier otra asignatura del plan de estudios.

La gama de métodos de enseñanza que se han de utilizar en una prescripción curricular para la tecnología estará, desde luego, condicionada por la finalidad y el ámbito de la provisión propuesta. Una gama adecuada se encontraráa dentro de lo que Shulman (1987) llama «acción y razonamiento peđagógico" para la prescripción. Ello consiste en seis elementos:

* La comprensión del profesor del contenido del tema de la prescripción. Como ya se ha comentado, éste será un tema importante en la formación de profesores. Cuanto más orientado hacia el futuro esté el programa, más tendrán que aprender los profesores.

* Formas de transformar el contenido del tema para que se relacione con lo que los estudiantes ya saben sobre tecnologia. 
* Los métodos de enseñanza que se pueden utilizar. Según experiencias recientes, parece ser que el enfoque basado en proyectos debería tencr un papel destacado en la enseñanza del aspecto técnico de Pacey (1983), mientras que la habilidad de manejar un tema controvertido scrá importante en los aspectos culturales y de organización.

* Lá evaluación de la cnseñanza y la valoración del aprendizaje de los estudiantes. Todos los profesores controlan continuamente su enseñanza y procuran mejorarla. La práctica de la evaluación formal de la enscĩanza difiere demasiado entre los países para que se puedan comentar aquí con garantías. Dada la centralización de las valoraciones en muchas perspectivas de planes de estudios actuales, es sorprendente que no se ha prestado una atención especial a las especiales circunstancias de la tecnología, aunque se ha llevado a cabo alguna tarea innovadora (APU 1991).

* Una reflexión sobre lo que se ha conseguido. La creciente toma de conciencia sobre el concepto de la reflexión práctica (Schon 1983) hace que parezca más probable que esto se dé más sistemáticamente en la tecnología.

* La revisión de la provisión. Es vital que la prescripción y la práctica no se anquilosen: la tecnología tiene un elemento inherente de crecimiento y cambio en su constitución que se ha de ver reflejado en la educación tecnológica.

\section{EL FUTURO DE LA EDUCACIÓN TEC- NOLÓGICA}

Se ha descuidado la educación tecnológica durante demasiado tiempo dentro de la educación formal. Una combinación de circunstancias estátlevando actualmen- te a un aumento del interés y la actividad. Si esta actividad ha de ser válida, se ha de basar en la investigación de las diferentes naturalezas de la tecnología y la educación tecnológica. A pesar de que se han detectado programas muy amplios (Gilbert 1992), sólo se han llevado a cabo unos cuantos estudios detallados de la clase (Jones y Carr 1993b). Si esto no se soluciona, la innovación de inspiración política, el enemigo de un auténtico avance educativo, predominará. Es importante que el alcance de la educación tecnológica proporcionada sea tan amplio como sea posible: la educación en tecnología ha de ser el objetivo de todos los sistemas educativos. Una orientación hacia los temas del medio ambiente le darán la base racional más fuerte para una aportación al futuro de la humanidad.

La tendencia general del pensamiento educativo durante la última década ha destacado una serie de ideas relacionadas entre sí. Una escolaridad que ofrece buenas oportunidades para una enseñanza constructiva y un aprendizaje eficaz y que contribuya considerablemente al desarrollo de los estudiantes a largo plazo se basa en:

* temas que son, o fácilmente se pueden considerar, importantes para los intereses y preocupaciones de los estudiantes;

* unas relaciones válidas y claras con formas de conocimientos utilizados en la comunidad (adulta) general;

* la investigación que tiene una orientación hacia el trabajo por proyectos, se fundamenta en las cuestiones propuestas por los estudiantes y tiene en cuenta la recogida y la evaluación de información procedente de varias fuentes;

* capacidades desarrolladas y reconocidas de los estudiantes.

La educación tecnológica tiene la posibilidad de cumplir todos estos criterios. Una reflexión sobre el discurso anterior pude ayudar a desarroltar este potencial en todo el mundo.

\section{NOTA}

Ponencia presentada en el IV Congreso Internacional sobre Investigación en la Didácticá de las Ciencias y de las Matemáticas, celebrado en Barcelona los días 13 al 16 de septiembre de 1993. Ha sido traducida del inglés y revisada por Mariona lispinet. 


\section{REFERENCIAS BIBLIOGRÁFICAS}

AAAS, 1989. Science for all Americans: Summary Report. (American Association for the Advancement of Science: Washington, DC)

AGPS, 1992. Technology for Australian Schools. (Austratian Government Publishing Service: Canberra).

APU, 1992. The assessment of performance in design and technology. (Schools Examination and Assessment Council: Londres).

BELL, B., y GILBERT, J. (en preparación). Teacherdevelopment: a model from science education.

BLANDOW, D, y DYRENFURTH, M. (eds.), 1992. Technological literacy, competence and innovation in human resource development. Proccedings of an International Conference on Technology Education. (Weimar: Alemania).

BUDGETT-MEAKIN, C. (ed.), 1992. Making the future work. (Longmans: Londres).

DAVIES, T., GILBERT, J. y DILLON, P., 1991. The Six Counties Technology Project: Management Folder. (Longman: Londres).

DILLON, P. y DAVIES, T., 1993. Real contexts for design and technology: an evaluation of the Six Counties Technology Flexible Learning Project. Presented at the IDATER conference, Lougborough, UK, September 1993.

EGGLESTON, I., 1992. Teaching design and technology. (Open University Press: Buckingham).

FULLAN, M., 1991. The new meaning of educational change. (Cassell: Londres).

GARDNER, H., 1985. Frames of Mind. (Basic Books: Nueva York)

GARDNER, P., PENNA, C. y BRASS, K, 1990. Technology and science: meanings and educational implications, The Australian Science Teachers Journal, 36(3), pp. 23-28.

GILBERT, J., 1992. The interface between science education and technology education, International Journal of Science Education, 14(5), pp. 563-578.

JONES, A. y CARR, M., 1993a. Toward's technology education. Centre for Science and Mathematics Education Research, University of Waikato. (Hamilton: Nueva Zclanda).

JONES, A. y CARR, M., 1993b. Analysis of student technological capability. Centre for Science and Mathematics Education Research, University of Waikato. (Hamilton: Nueva Zelanda).

JUMA, C. y SAGOFF, M., 1992. Policies for technology, en Dooge, J., Goodman, G., La Rivière, J., Martín-Lefebre, J., O'Riordan, J. y Praderie, F. (eds.), An agenda of science for environment and development in the $2 /$ st. century, pp. 265281. (Cambridge University Press: Cambridge).
KIRKUP, G. y KELLER, L., 1992. Inventing women: science, fechnology and gender. (Polity Press: Cambridge).

LAYTON, D., 1988. Revaluing the $\mathrm{T}$ in STS, Intemational Journal of Science Education, 10(4), pp. 367-378,

LAYTON, D., 1990a. Science education and the new vocationalism, en Jenkins, E. (ed.), Policy issues and school science, pp. 52-62. (School of Education, University of Leeds: Leeds)

LAYTON, D., 1990b. Inarticulate science. (Department of Education, University of Liverpool: Liverpool).

LAYTON, D., 1993. Technology's challenge to science education. (Open University: Buckingham).

MACKAY, $H_{n}, 1991$. Technology as an educational issue social and political perspectives, en Mackay, $\mathrm{H}$., Young, $M$ y Beynon, J., (eds.), Understanding technology in education, pp. 1-12. (Falmer: Londres).

MARGOLIS, J., 1984. Three conceptions of technology: satanic titanic, human, en Durbin, P. (ed.), Research in Philosophy and Technology, Vol. 7, pp. 145-176.

MEEKE, N., 1989. The concept of key technologies. (Further Education Unit: Londres).

MEDWAY, P., 1989. Issues in the theory and practice of technology education, Studies in Science Educarion, 16, pp. $1-24$.

MILLAR, R. y DRIVER, R., 1987. Beyond Processes, Studies in Science Education, 14, pp. 33-62.

PACEY, A., 1983. The culture of technology. (Blackwell: Oxford).

RAPP, F., 1989. Introduction: general perspectives on the complexity of philosophy of technology, en Durbin, P. (ed.), Philosophy of technology: practical historical and other dimensions, pp. ix-xxiv. (Kluwer: Dordrecht).

SCHILLING, M., 1988. Science, Technology or Science and Technology, en Institute of Physics (ed.). Physics and technology: interactions in education, pp. 17-22. (Institute of Physics: Bristol).

SCHON, D., 1983. The Reflective Practitioner. (Temple Smith: Londres).

SHULMAN, L., 1987. Knowledge and teaching: foundations of a new reform, Educational Review, 57(1), pp. 1.22.

SILVERSTONE, R., 1991. The body electric, The Times Higher Education Supplement, 2, pp. 13-15.

TOLBA, M., 1992. Saving our planet: challenges and hopes. (Chapman and Hall: Londres).

WCED (World Commission on Environment and Development), 1987. Our common future (Oxford University Press: Oxford). 